Journal of Science
$\mathrm{http} / / /$ dergipark.gov.tr/gujs

\title{
Computer-Aided Prediction for Printable Density Limits of Additively- Manufactured Direction-Wise Stretch-Dominated Strut-Based Lattice Structures
}

\author{
Niyazi TANLAK * (in \\ Turkish Aerospace Industries Inc, Teknopark Istanbul 34906, Pendik Istanbul Turkey
}

Article Info

Received: 21 Nov 2020

Accepted:30 Mar 2021

\section{Keywords \\ Cellular structures \\ Lattice \\ Additive manufacturing \\ Powder bed fusion \\ Printable relative density}

\begin{abstract}
When topologies of the bending-dominated lattices are strengthened by introducing a strut being oriented in one cubic direction, the lattice structures may behave like a stretch-dominated one in that direction. Because of this potential, they are of interest to engineers demanding anisotropic advanced materials. But their manufacturability is as important as the mechanical advantage they can present. To manufacture these lattice structures, additive manufacturing methods like powder bed fusion are widely used. Yet, there are limits for printing these structures. In this study, taking machine precision and powder lump size relative to the unit-cell size as main factors, the printable density range was found for the first-time for the lattice structures strengthened by adding a strut in one direction. Results indicated that the printable relative density range shifted upward in comparison to lattices which were not strengthened.
\end{abstract}

\section{INTRODUCTION}

A solid-foam is a structure consisting of a network of solid and gas-filled pores. By the time a foam-like structure is formed out of a solid, the single-valued properties of that solid are extended. Those properties are the strength, stiffness, electrical/thermal conductivity, and diffusivity, and so forth [1]. Because of this property extension, foams are used in many advanced practices like biomedical and aerospace engineering. The property extension can be made more controllable by the introduction of structured foams, a unit-cell (or a network of struts) repeating itself throughout a domain. Yet, structured foams are known to be hard to be manufactured by using conventional tools. At this point, additive manufacturing (AM) offers what was not possible in the manufacturing of them previously.

Contrary to the common belief that a cellular structure is always printable, structured foams face printing problems. The printable density limits of unit-cell (a.k.a. lattice) types are not fully known. In powder bed fusion methods, printability of a structured foam depends on unit-cell shape/topology [2-9], relative density of a lattice (i.e. volume fraction) [2-5,7,8,10-13], strut inclination [14-20], cell size [5], powder particle size [5,21-23], powder material [3,4,8,10,16,17,24], machine precision [2,5,10,11,25,26], laser power [3, 4, 10, 23, 25-31], laser spot size [10, 22, 26, 32], laser scanning speed [3, 4, 25, 28-31, 33], layer thickness $[10,30,33]$, hatch spacing [22, 29], building orientation [15, 34-36], part thickness [12] and 
time [37]. Among these factors, machine precision together with unit-cell size are the main factors for low relative density limit while powder lump size is the main factor defining high relative density limit.

An important point worth mentioning here is that entrapment of powder within an inclusion still allows the manufacture. But the mass of such structured-foam will be higher than that of the intended one, which is taken to be improper in this study, and also against the definition of foams.

Engineers generally demand materials exhibiting stronger behavior in one direction than the others. Composite materials are a typical example of this. Lattices can also offer such kind of property. Some researchers [38] reported that some lattice types show stretch-dominated behavior despite appearing to violate the Maxwell criterion [39-41], which is taken in the literature as the necessary condition to show that a lattice is stretch-dominated if $M=0$ in the equation below in three-dimensional space

$$
M=b-3 j+6=s-m
$$

where $b$ is the number of struts; $j$ designates frictionless joints; $s$ counts the number of states of self-stress and $m$ counts the number of mechanisms.

When topologies of the bending-dominated lattices are strengthened by adding a strut into the unit-cell (generally, it is stated as Z-strut in the literature), the lattice structures may behave like a stretch-dominated one, resulting to be stiffer, stronger and in need of bigger loads to cause deformation and yielding [42]. This implies that the lattice topologies which is defined, according to Maxwell criterion, as bendingdominated like BccZ and FccZ (See Figure 1) can exhibit stretch-dominated behavior based on the load's orientation with respect to the lattice [43]. Tanlak et al. [5] investigated the printable limits of some wellknown strut-based lattice structures like Bcc, Fcc, and so on. However, the printability of direction-wise stretch-dominated strut-based lattice structures was an open question. As they have promising potential, their printability should be investigated to provide practical tools to designers when they are manufactured by using powder bed fusion techniques. Yet, this work can cover other AM methods.

\begin{tabular}{|cl|}
\hline AM & Additive manufacturing. \\
C.P. & The cross-plane perpendicular to $\gamma$ at SP. \\
SP & Separation point. \\
$A_{\min }$ & Powder cross-sectional area. \\
$\mathrm{I}$ & The interval over which $\gamma$ defined. \\
$I_{v}$ & The interval over which $\Omega_{v}$ defined. \\
$\mathrm{L}$ & The cell size of a lattice. \\
$L_{\text {strut }}^{\text {tot }}$ & Total strut length. \\
$n_{v o i d}$ & Number of inclusions within the unit-cell. \\
$\mathrm{P}$ & Any point. \\
$R^{2}$ & The coefficient of determination. \\
$\mathrm{r}$ & Strut radius. \\
$\bar{r}$ & Relative strut radius. \\
$r_{h}^{\prime}$ & The strut radius value corresponding to $\rho_{\text {high }}$ for infinitesimally thin powder case. \\
$r_{h}$ & The strut radius value corresponding to $\rho_{\text {high }}$ for finite-size powder case. \\
$\overline{r_{h}^{\prime}}$ & The relative strut radius value corresponding to $\rho_{\text {high }}$, i.e. $\overline{r_{h}^{\prime}}=\frac{r_{h}^{\prime}}{L}$. \\
$r_{l}$ & The strut radius value corresponding to $\rho_{\text {low }}$ for finite-size powder case. \\
$r_{l u m p}$ & The radius corresponding to $V_{p}$. \\
$r_{\text {min }}$ & The minimum printable radius. \\
$\mathrm{t}$ & Lattice type. \\
$V_{p}$ & The volume of the powder particle lump. \\
$\gamma$ & A space curve. \\
$\kappa$ & The curvature of a curve. \\
$\kappa(\gamma)$ & Curvature of the space curve $\gamma$. \\
$\rho$ & Relative density. \\
$\rho^{\text {corr }}$ & Correction term in relative density. \\
\hline
\end{tabular}




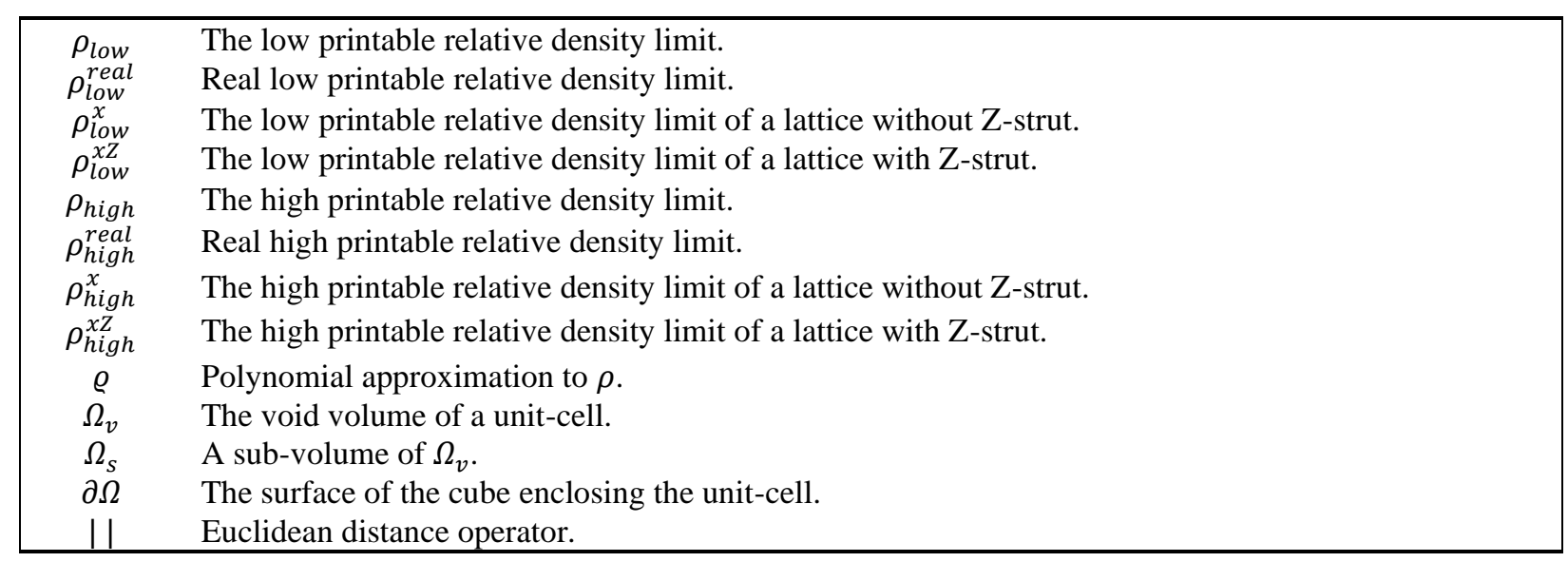

\section{MATERIAL}

In this study, the unit-cell types presented here are the modified versions of well-known lattices, by introducing Z-strut, which are depicted with cyan color in Figure 1. Bcc (Body centric cubic), Fcc (Face centric cubic), Octahedron, Cubic are the basic unit-cells, which are depicted with violet, orange, olive, black colors, respectively. The names of the basic unit-cells were used because of their similarities to the respective atomic crystals. Here, the atoms in the crystals were replaced with struts connecting center points of atoms. The other unit-cells in Figure 1 were the combinations of these basic unit-cells. But the lattices in Figure 1 were selected in outline; this was by no means an exhaustive list. Each unit-cell was considered to be confined within cubic space. To make lattices exhibit stronger behavior in one direction, a single strut spanning along $\mathrm{z}$-direction was appended right into the center of the unit-cell. By doing so, these lattices gained the advantage of minimum interference to neighbors. They did not touch any edges but only two faces of their neighbors.

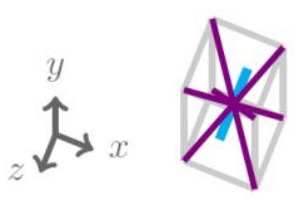

BccZ

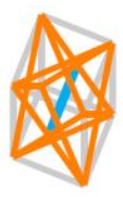

FccZ

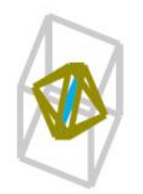

OctahedronZ

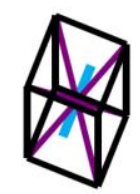

CubicBccZ

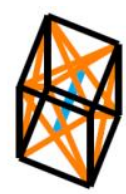

CubicFecZ

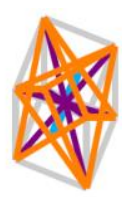

BccFccZ

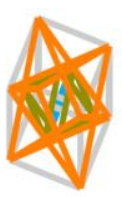

OctetZ

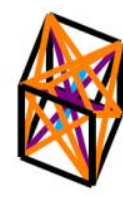

CubicBccFccZ

Figure 1. Unit-cell library

Machines are bounded in terms of precision, leading to the smallest printable radius, which in turn, leads to the low printable density limit for strut-based lattices. Equation (2) expresses the numerical prediction of the low printable density limit of lattice topologies in Figure 1 for powder bed fusion by Tanlak et al. [5] as follows:

Minimize

Such that

$$
\begin{aligned}
& \rho=\rho\left(r, t, L, r_{\min }\right) \\
& \text { 1) } 0<r \leq L \\
& \text { 2) } \quad \forall t \in T \mid T=\{\text { BccZ, FccZ, OctahedronZ, CubicBccZ, CubicFccZ, } \\
& \text { BccFccZ, OctetZ, CubicBccFccZ }\} \\
& \text { 3) } \mathrm{L}>0 \\
& \text { 4) } 0<r_{\text {min }} \leq L
\end{aligned}
$$

where $\rho$ is the relative density; $r$ is the strut radius; $t$ is the lattice type; $L$ is the lattice size; $r_{\min }$ is the minimum printable radius. $T$ is the set of lattice types.

Equation (2) states that its objective was to find the lower printable density for a given lattice type and size, and machine precision. The values of strut radius should be bigger than zero and should be smaller than $L$ (See Equation (1)). Equation (2) stated the lattice library studied. Equation (2) enforced the length of the unit-cell to be a positive real number. Finally, machine precision was dictated to be a positive number which is smaller than $L$ by Equation (2). 
On the other hand, powder lump size and unit-cell size were the main factors defining the high relative density limit. The powder in the bed, in no doubt, had a size. On top of that, due to the spatter landing to the unintended regions or loose particles' partial sintering [18], the leftover particles could create lumps during the process. This made their effective radius even bigger. As mentioned before, entrapment of powder would make the mass of the structured-foam higher than that of the intended one, which was considered to be improper.

The numerical prediction of the maximum printability density of lattice topologies in Figure 1 for powder bed fusion was expressed mathematically by Tanlak et al. [5] as:

Maximize

$\rho=\rho\left(r, t, L, r_{\text {lump }}\right)$

Such that

1) $0<r \leq L$

2) $\forall t \in T \mid T=\{$ BccZ, FccZ, OctahedronZ, CubicBccZ, CubicFccZ, BccFccZ, OctetZ, CubicBccFccZ\}

3) $\mathrm{L}>0$

4) $0<r_{\text {lump }} \leq L$

5) $n_{\text {void }}=1$

6) $\exists \Omega_{s} \mid \Omega_{s}=\left\{P \in \Omega_{v} \wedge|P, \gamma| \leq r_{\text {lump }}\right\}$

7) $\gamma: I \rightarrow \boldsymbol{R}^{3} \& \gamma \subset \Omega_{v} \& I=I_{v}$

8) $\min \left(\frac{1}{\kappa(\gamma)}\right) \geq r_{\text {lump }}$

where $r_{\text {lump }}$ is the powder lump radius; $n_{\text {void }}$ is the number of inclusions within the unit-cell; $\Omega_{v}$ is the void volume of a unit-cell; $\Omega_{S}$ is a sub-volume of $\Omega_{v} ; P$ designates any point; $\gamma$ is a space curve; $I$ is the interval over which $\gamma$ defined; $I_{v}$ is the interval over which $\Omega_{v}$ defined; $\kappa(\gamma)$ is the curvature of the space curve $\gamma$.

Equation (3) states that its objective was to find the upper printable density for a given lattice type and size, and machine precision. The first four constraints stated the same things in Equation (2) only with the exception of powder lump size, $r_{\text {lump }}$ replaces the minimum printable radius. In order to make the intended and the resultant volume fraction of any cellular structure the same, the unused powder should be evacuated after printing. The lattice had to have a monolithic void volume (See the fifth constraint in Equation (3)). At this point, one could assume that a single lattice with an independent void was printable if those inclusions reach the boundary of the lattice. But these independent inclusions ended up to be closed cavities trapping powder when that lattice was surrounded by others. That was why $n_{\text {void }}$ was forced to be unity. Yet, to enable the powder to pass through, the void volume had to provide enough room inside. This had to be a single void subspace having branches as thick as powder lump at minimum. To define the mentioned void subspace, a space curve was employed over the same interval which the unit-cell was defined over (See Equation (6)). By constraining the minimum distance to the formulation of $\Omega_{s}$, a pipe-like volume having a thickness of $r_{l u m p}$ at least was formed throughout the interval. To ensure that the void volume reached the boundary of the cubic envelope so that the powder could be evacuated, a sub-volume, $\Omega_{S}$, within the void volume whose interval was the same as the interval of the void volume defined over was forced to exist in the constraint 6 of Equation (3). The curvature of the space curve (the center-line of the pipe-like void volume), $\gamma$, matters, as a curve having a smaller radius of curvature at any point than the powder lump radius was actually an obstacle blocking the powder's flow. That is why it was restrained as defined in Equation (8).

\section{METHOD}

Tanlak et al. [5] proposed a methodology of dividing the problem into two cases: Infinitesimal powder and finite powder case. At first instance, the problem was taken into consideration by assuming the bed full of infinitesimally thin powder and the printer having infinite-precision. This assumption would provide the ideal case and it will be the pivot point to attack the problem of finite size powder and finite-precision production. 


\subsection{Infinitesimally Thin Powder \& Infinite Precision Production Problem}

In this scenario, the powder was assumed to be infinitesimal in comparison to the cell size and the machine used has infinite precision. Therefore, any shape was within the printable realm. The powder could be taken out if the void volume was monolithic and reached the boundary of the unit-cell. Hence, the cell size ceased to be a decisive parameter for $\rho_{\text {low }}$ and $\rho_{\text {high }}$.

$\rho_{\text {low }}(r, t)$, would be the smallest positive quantity, $\varepsilon$. Thus, only $\rho_{\text {high }}(r, t)$ would be calculated in this case. Therefore, the problem stated in Equation (3) became as below:

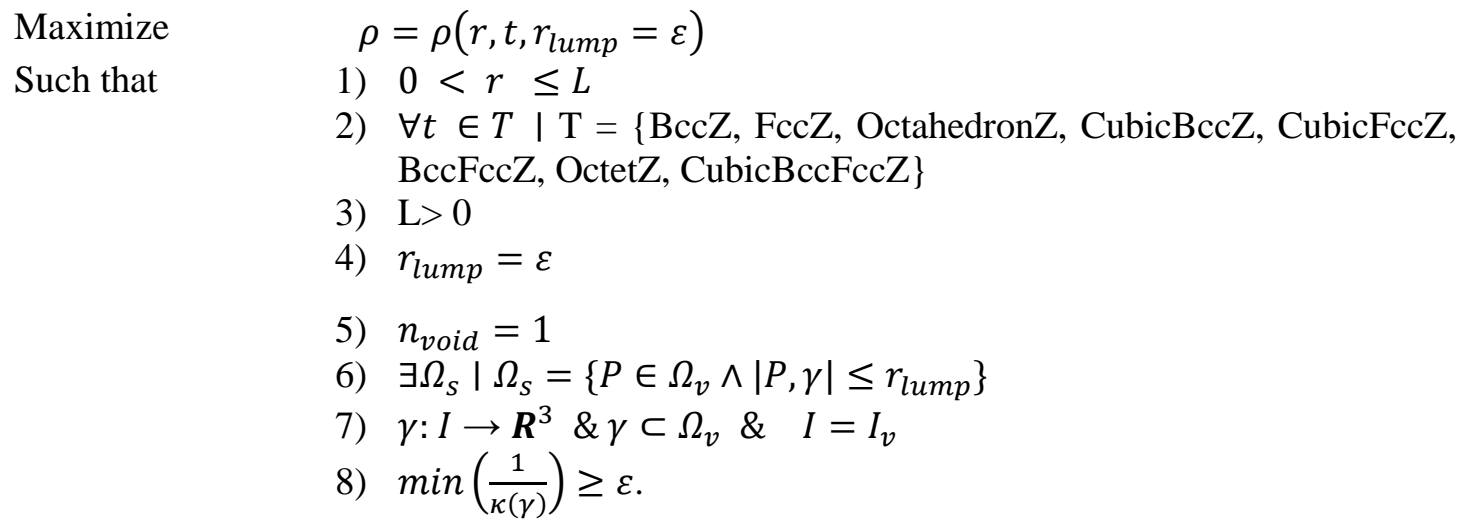

CAD software used (the preprocessor of Abaqus) [44] can create geometric entities having an edge length as small as $10^{-6}$. For this reason, $\varepsilon$ was taken to be $10^{-5}$. $n_{\text {void }}$ was an integer-valued piece-wise function that depended on $r$ (See Figure 2 for a typical change). In other words, after some critical value of relative strut radius, individual void rooms began to form. In order to reach the high printable density limit, the maximum $r$ satisfying $n_{\text {void }}=1$ needed to be found, which was $r_{h}^{\prime}$. To find $r_{h}^{\prime}$, the modified bisection method [5] is adopted.

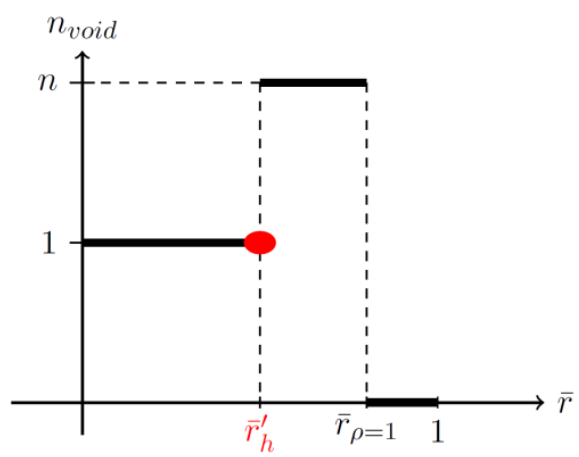

Figure 2. Typical change of $n_{\text {void }}$ with respect to $\underline{r}$ of a strut-based lattice [5]

\subsection{Finite Size Powder \& Finite Precision Production Problem}

\section{Computation of $\rho_{\text {low }}$}

Because the machine used in this case had finite precision, the minimum printable relative density would be bigger in comparison to the infinite precision case. Calculating the relative density for the specified machine precision was the only thing to find the low relative density for a specified case. Therefore, one needed to plug $r=r_{\min }$ to Equation (2). Calculating relative density based on relative radius, $\rho=\rho(\bar{r})$, and fitting the data to a polynomial, $\varrho$, first made the calculation of $\rho_{\text {low }}$ easier. Then, the low relative density limit could be found via plugging dimensionless machine precision value, $\frac{r_{\min }}{L}$, to $\varrho$. In plain mathematics, it can be as follows: 


$$
\rho_{\text {low }}=\varrho\left(\bar{r}=\frac{r_{\text {min }}}{L}, t, L\right) .
$$

\section{Computation of $\rho_{\text {high }}$}

Here, the powder had a size, the high limit of the printable relative density would be lower in comparison to the infinitesimal powder case. To put it in another way, the printable density window of a lattice structure would be smaller than the infinitesimal powder problem in the real world.

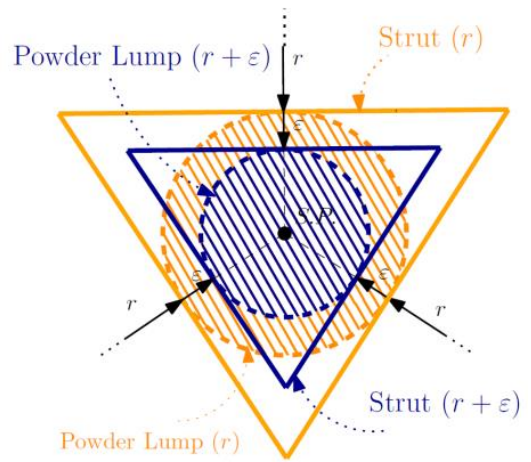

Figure 3. Typical cross-sectional shape change of the void volume around a bottleneck

In infinitesimal powder and infinite precision production case, point contacts kept the void volume together as SP because of the fact that the powder was a point. As the strut radius changed, void cross-section altered like polygons nested within each other while keeping their orientation the same as depicted in Figure 3. Taking courage from this fact, as Tanlak et al. [5] suggested, the critical strut radius for the infinitesimal powder case, $r_{h}^{\prime}$ was lowered, as much as powder size, $r_{\text {lump }}$, to find the high printable density limits. In plain mathematics, $r_{h}\left(V_{p}=\frac{4}{3} \pi r_{\text {lump }}^{3}\right)=r_{h}^{\prime}\left(V_{p}=\frac{4}{3} \pi \varepsilon^{3}\right)-r_{\text {lump }}$. Then, one could find the maximum printable volume fraction as follows:

$$
\rho_{\text {high }}=\varrho\left(\frac{r_{h}^{\prime}\left(v_{p}=\frac{4}{3} \pi \varepsilon^{3}\right)}{L}-\frac{r_{l u m p}}{L}\right) .
$$

To objectify the proposed methods in this work, the preprocessor of Abaqus software, was employed with Python language.

\section{THE RESEARCH FINDINGS AND DISCUSSION}

Relative density of each lattice topology was plotted as a function of $\bar{r}$ in Figure 4 . The corresponding data was approximated via cubic polynomials to provide an easy-to-use tool for designers, whose coefficients were tabulated in Table 1. 


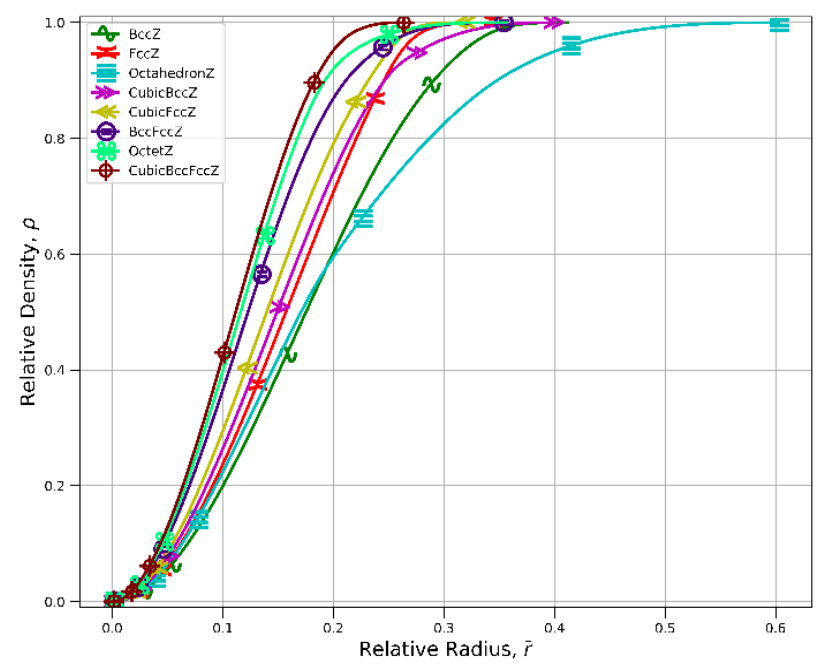

Figure 4. Relative density change as a function of relative strut radius

As can be seen from Figure 4, CubicBccFccZ was on the left-hand side while BccZ was on the right-hand side in comparison to other curves. This meant that CubicBccFccZ was the quickest at reaching highdensity values while $\mathrm{BccZ}$ was the slowest. One moment of consideration would reveal that those having more struts within, were the ones reaching full density quicker. For very low relative radius values, the volume fraction followed almost quadratic relation, i.e. $\rho \simeq L_{\text {strut }}^{\text {tot }} r^{2}$. However, this trend could not be sustained because of the merging of the struts. That is why the curves exhibited saturation as their relative radius increased. Yet, those on the left-hand side would need thinner struts to reach the same volume fraction. This creates printability issues for the lattice having more struts for low volume fractions because of inadequate machine precision.

Table 1. Coefficients of the polynomials, $\varrho$, approximating to $\rho$ until printable relative density

\begin{tabular}{|l|c|c|c|}
\hline Unit-cell & $\overline{\mathrm{r}}^{3}$ & $\bar{r}^{2}$ & $R^{2}$ \\
\hline BccZ & -47.01 & 24.40 & 0.999 \\
\hline FccZ & -61.11 & 29.89 & 0.999 \\
\hline OctahedronZ & -74.27 & 29.83 & 0.999 \\
\hline CubicBccZ & -79.19 & 34.34 & 0.999 \\
\hline CubicFccZ & -97.29 & 39.22 & 0.999 \\
\hline BccFccZ & -147.70 & 51.26 & 0.999 \\
\hline OctetZ & -166.99 & 56.49 & 0.999 \\
\hline CubicBccFccZ & -185.79 & 60.89 & 0.999 \\
\hline
\end{tabular}

\subsection{Infinitesimally Thin Powder \& Infinite Precision Machine Production Problem}

The high relative density and the corresponding critical relative radius values are provided in Table 2 . OctahedronZ was the one having the lowest $\rho_{\text {high }}$ while BccZ had the highest $\rho_{\text {high }}$ as occurred in Octahedron lattice reported by Tanlak et al. [5]. The high relative density limit of lattice topologies studied here showed an increase except for OctahedronZ and OctetZ. But the differences were dim.

Table 2. The high relative density and the corresponding critical relative radius

\begin{tabular}{|l|l|l|l|l|l|}
\hline Unit-cell & \multicolumn{1}{|c|}{$\rho_{\text {high }}$} & \multicolumn{1}{c|}{$\bar{r}_{h}^{\prime}$} & \multicolumn{1}{c|}{$\bar{r}_{h}^{\prime}$} & \multicolumn{1}{c|}{$\rho_{\text {high }}$} & Unit-cell \\
\hline BccZ & 0.9906 & 0.3553 & 0.3553 & 0.9886 & Bcc \\
\hline FccZ & 0.9702 & 0.2706 & 0.2887 & 0.9073 & Fcc \\
\hline OctahedronZ & 0.5703 & 0.1913 & 0.2024 & 0.5849 & Octahedron \\
\hline CubicBccZ & 0.9264 & 0.2588 & 0.2588 & 0.8902 & CubicBcc \\
\hline CubicFccZ & 0.8181 & 0.2071 & 0.2071 & 0.7365 & CubicFcc \\
\hline BccFccZ & 0.9069 & 0.2142 & 0.2142 & 0.9065 & BccFcc \\
\hline OctetZ & 0.8969 & 0.1913 & 0.2041 & 0.9073 & Octet \\
\hline CubicBccFccZ & 0.9673 & 0.2071 & 0.2071 & 0.9660 & CubicBccFcc \\
\hline
\end{tabular}


For all lattice topologies, CAD models of void and solid volumes are shown as isolated from each other as well as in assembled form for $\rho_{\text {high }}$ in Table 3 . As can be seen from the table, the void volume (depicted as blue in Table 3) gets so thin that it keeps its contact with its rest with a point contact. Those points are named as separation points (SP) in this article. SP's of BccZ, CubicFccZ, CubicBccFccZ were on the faces of the cubic enclosing envelope while SP's were inside for the rest. In Table 3, it is hard to see that the void volumes of CubicFccZ and CubicBccFccZ reach their boundary when the respective void and solid volumes are assembled. However, one moment look at the isolated void volumes of CubicFccZ and CubicBccFccZ in Table 3 will reveal that the void volumes maintain their point connection with their boundary, which is the sufficient condition for evacuating the infinitesimal powder out.

Table 3. CAD models of void and solid volumes of unit-cells when they reach their respective $\rho_{\text {high }}$

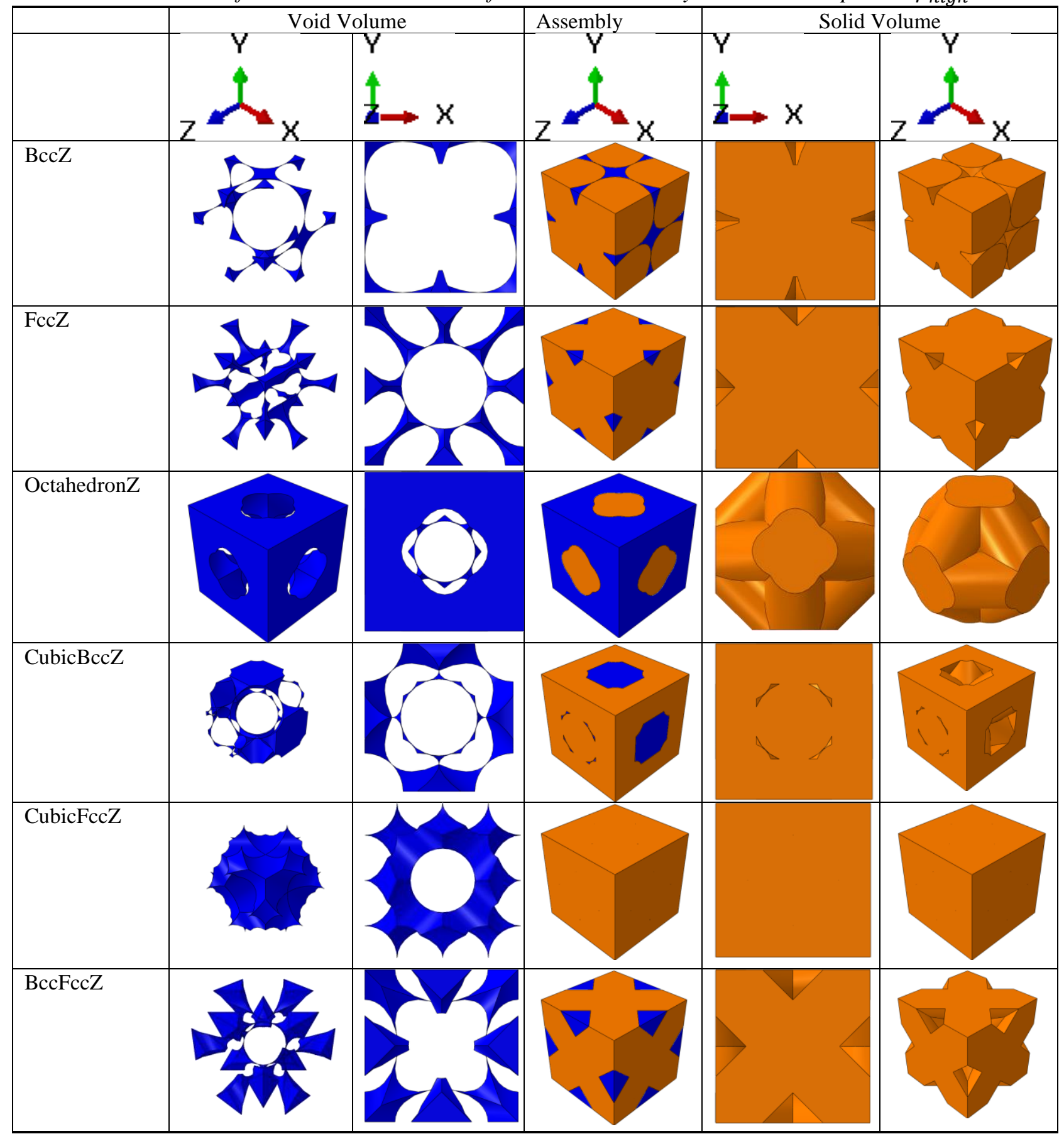




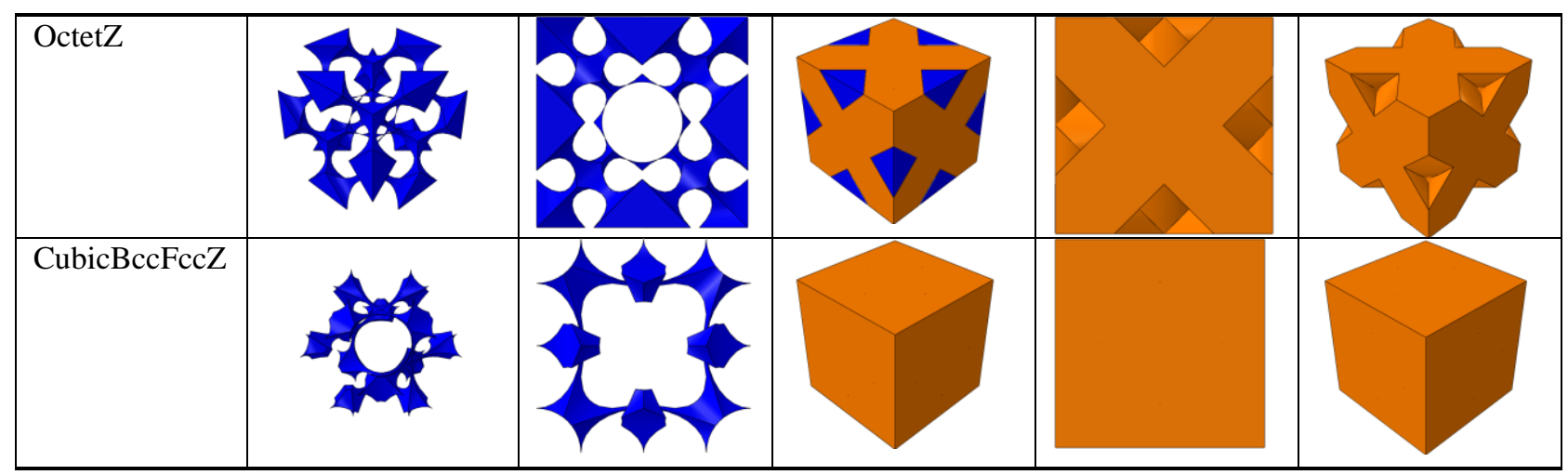

\subsection{Finite Size Powder \& Finite Precision Machine Production Problem}

Unlike the previous case, here we were subjected to machine precision and the powder size; ergo, there would be a lower as well as a high printable limit. In order to give an example, the lattices in range of 1-20 $m m(L)$, were assumed to be printed by using powder of $r_{\text {lump }}=0.12 \mathrm{~mm}$ in an additive manufacturing machine having of $r_{\min }=0.2 \mathrm{~mm}$. The results of that case are plotted in Figure 5 . As the cell size increased $(L)$, while all other factors remained the same, the printable range got closer to the infinitesimal case. When cell size decreased, the high and low limit curves of each unit-cell drew near to each other. In other words, the range of printable density got narrower, till the two curves intersected, which, in turn, meant that solely one density value was printable. From that point on, any unit-cell value was not printable. Smallest lattice in length and their corresponding relative density varied based on the lattice type. Among all the lattice types studied, the smallest in length to be printable was BccZ with $0.9 \mathrm{~mm}$ in length and the relative density of 0.69. Although the number of struts in BccZ and OctahedronZ were the same, BccZ had the widest printable density window while OctahedronZ had the narrowest. This was because of the unit-cell topology.

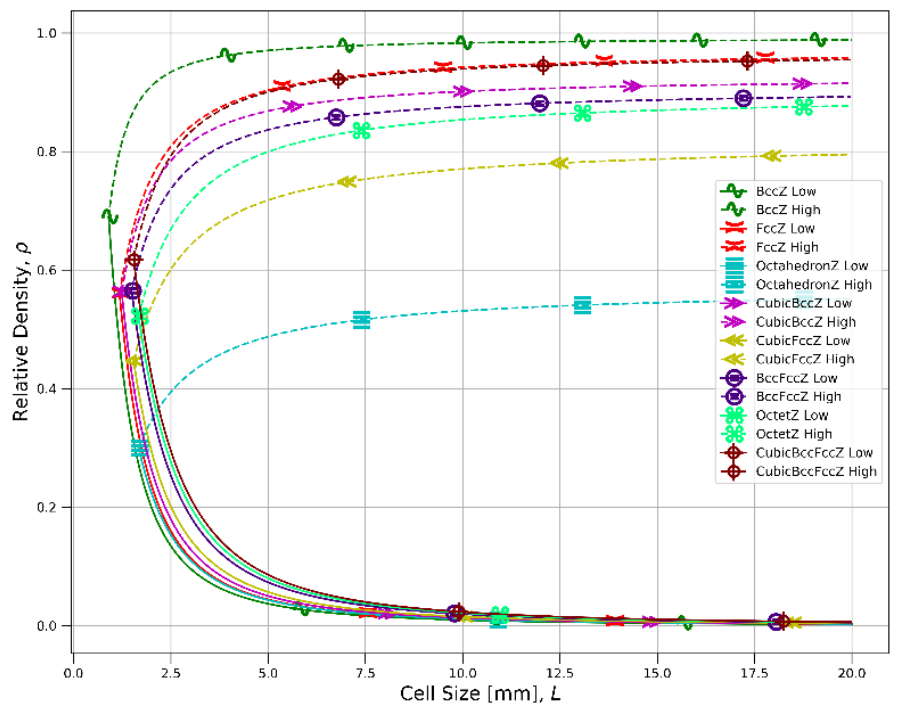

Figure 5. Lower and higher printable density limits for $r_{\min }=0.2 \mathrm{~mm}$ and $r_{\text {lump }}=0.12 \mathrm{~mm}$ as a function of cell size

Since the combination of machine precision, powder size, unit-cell size was endless, it was necessary to build a dimensionless plot enabling to find the corresponding printability limits for any combination of $r_{\text {lump }}, r_{\min }$ and $L$. In Figure 6, such a plot was provided, in which $\frac{r_{l u m p}}{L}$ and $\frac{r_{\min }}{L}$ were put on abscissa together. But they were mutually independent of each other. The relative density values in Figure 6, were cut-off at the corresponding $\rho_{\text {high }}$ of each unit-cell. When $\frac{r_{\min }}{L}$ value got bigger, it may have meant that either a smaller lattice was printed by the same machine or the same lattice was printed by another machine having coarser precision. On the other hand, when $\frac{r_{l u m p}}{L}$ value got bigger, it may have meant that either 
printing a smaller lattice by using the same powder size or printing the same lattice by another coarser powder.

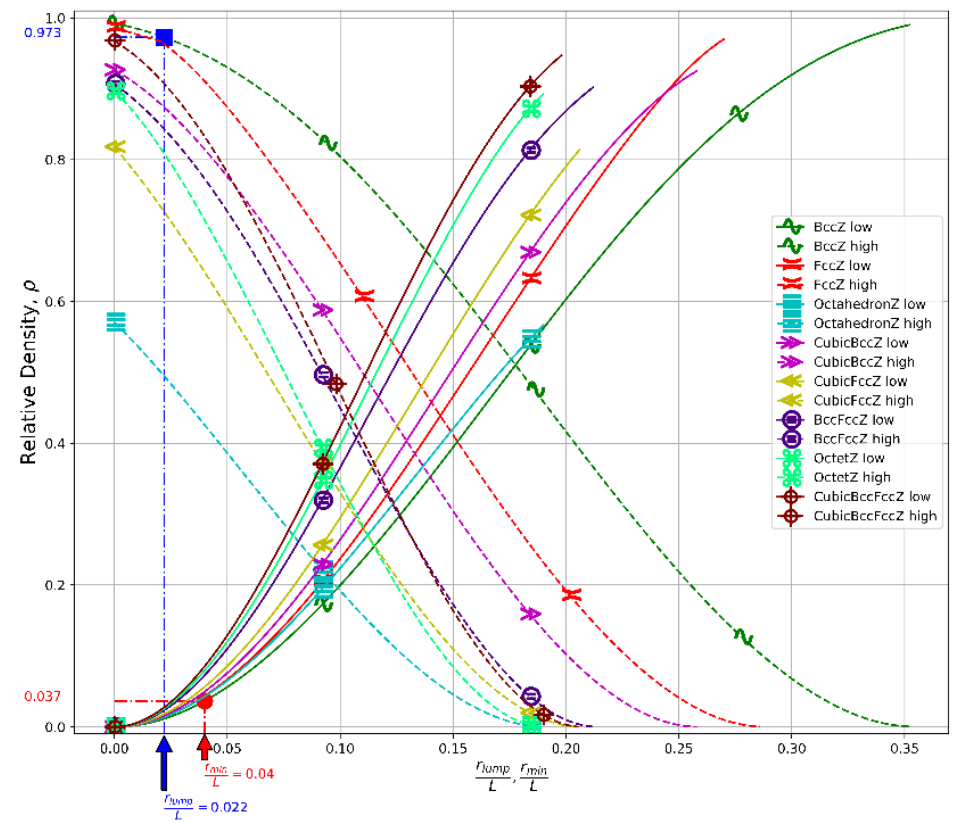

Figure 6. Printable density limits of lattice topologies in terms of dimensionless variables

In order to clarify how to use Figure 6, the printable window was found using $L=5 \mathrm{~mm}, r_{r m i n}=0.2$ $\mathrm{mm}$, and $r_{\text {lump }}=0.11 \mathrm{~mm}$ for BccZ lattice. The resultant range is [0.037, 0.973]. The corresponding values are marked in Figure 6 by red and blue dash-dots, respectively. The smaller the values of $\frac{r_{\min }}{L}$ and $\frac{r_{l u m p}}{L}$, the bigger the printable density windows. When $\frac{r_{\min }}{L}$ and $\frac{r_{\text {lump }}}{L}$ get bigger, these two limits draw closer. Inevitably, the two values will be the same. This means that only one relative density is possible to print. In other words, the low printable density limit cannot be bigger than the high one. If one finds that value for a particular case, it is safe to say that all other smaller values of $\frac{r_{\min }}{L}$ or $\frac{r_{\text {lump }}}{L}$ for a constant value of the other are safe to print. However, the number of cases is endless and $\frac{r_{\min }}{L}$ and $\frac{r_{l u m p}}{L}$ are mutually independent. One moment look would reveal that the low and the high relative density limits are mutually mirror-symmetric around $\frac{r_{h}^{\prime}}{L}$. Therefore, there will be lines regarding each lattice type. Based on Figure 6, one could deduce that there was a relationship for the high and low limits curves like below

$$
\frac{r_{\min }}{L}=\frac{r_{h}^{\prime}}{L}-\frac{r_{l u m p}}{L} .
$$

Equation (7) also states that the higher and the lower printable density limits were mutually mirrorsymmetric around $\frac{r_{h}^{\prime}}{L}$. Based on Equation (7), Figure 7 was created. This equation (also Figure 7) provides $\frac{r_{\min }}{L}$ for a given value of $\frac{r_{l u m p}}{L}$ where only one relative density is possible to print. Likewise, it provides $\frac{r_{\text {lump }}^{L}}{L}$ for a given value of $\frac{r_{\min }}{L}$. So, any value smaller than the resultant is printable while the plugged value is constant. In order to clarify how to use Figure 7, the printable $\frac{r_{\min }}{L}$ was found using $L=5 \mathrm{~mm}$, $r_{\text {lump }}=0.11 \mathrm{~mm}$ for BccZ lattice. The resultant range is [0,0.333]. The corresponding values are marked in Figure 7 by green dash-dots. 


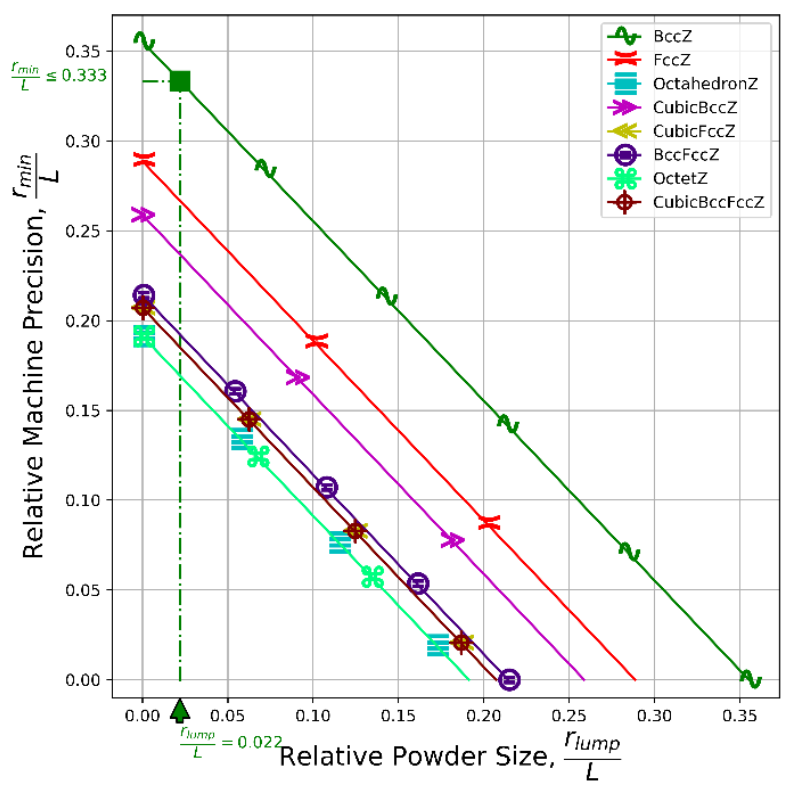

Figure 7. Printable zone regardless of relative density

\subsection{Comparison of Lattice Topologies with and without Z-Strut}

Lattices having fewer struts were advantageous at reaching low relative density because they needed thicker struts to reach the same density. This trend can be seen in Figures 5 and 6. Therefore, it means that they did not need a machine having finer precision. In order to clarify how much adding a Z-strut increased the low limit, a dimensionless plot stating the lower relative density difference between the lattices with $\left(\rho_{\text {low }}^{\mathrm{xZ}}\right)$ and without Z-strut $\left(\rho_{\text {low }}^{\mathrm{X}}\right)$ was generated in Figure 8 . The relative machine precision values in Figure 8 were cut-off at the corresponding $\rho_{\text {high }}$ of each unit-cell. Otherwise, the curves would end at zero. According to Figure 8 , the low limit increased except for the machine having infinitely small precision. The degree of that increase changed along with $\frac{r_{\min }}{L}$.

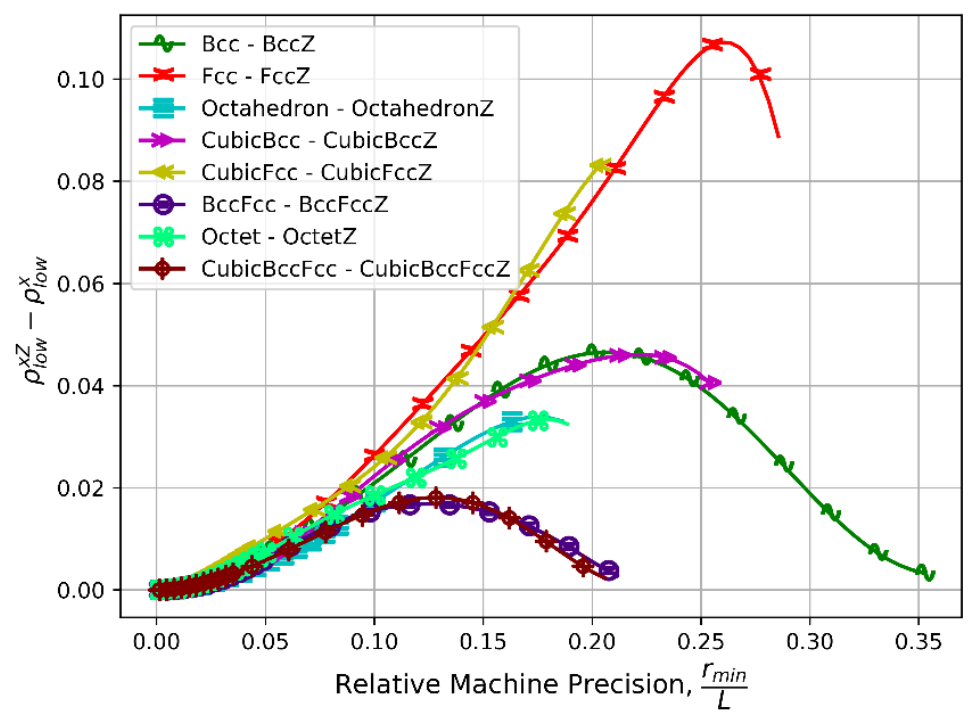

Figure 8. The difference between the lower printable density limits of lattice topologies with and without $Z$-strut in terms of the relative machine precision

On the other side of the coin, lattices having more struts were advantageous at reaching high relative density because they needed thinner struts to reach the same density. Therefore, this meant that there was more room for the powder to be evacuated. In order to clarify how much adding a Z-strut increased the high limit, a dimensionless plot stating the high relative density difference between the lattices with and without Zstrut was generated in Figure 9. The relative powder lump values in Figure 9 were cut-off at the 
corresponding $\rho_{\text {high }}$ of each unit-cell. Otherwise, the curves would start from zero. According to Figure 9, the high limit increased. The degree of that increase changed along with $\frac{r_{l u m p}}{L}$.

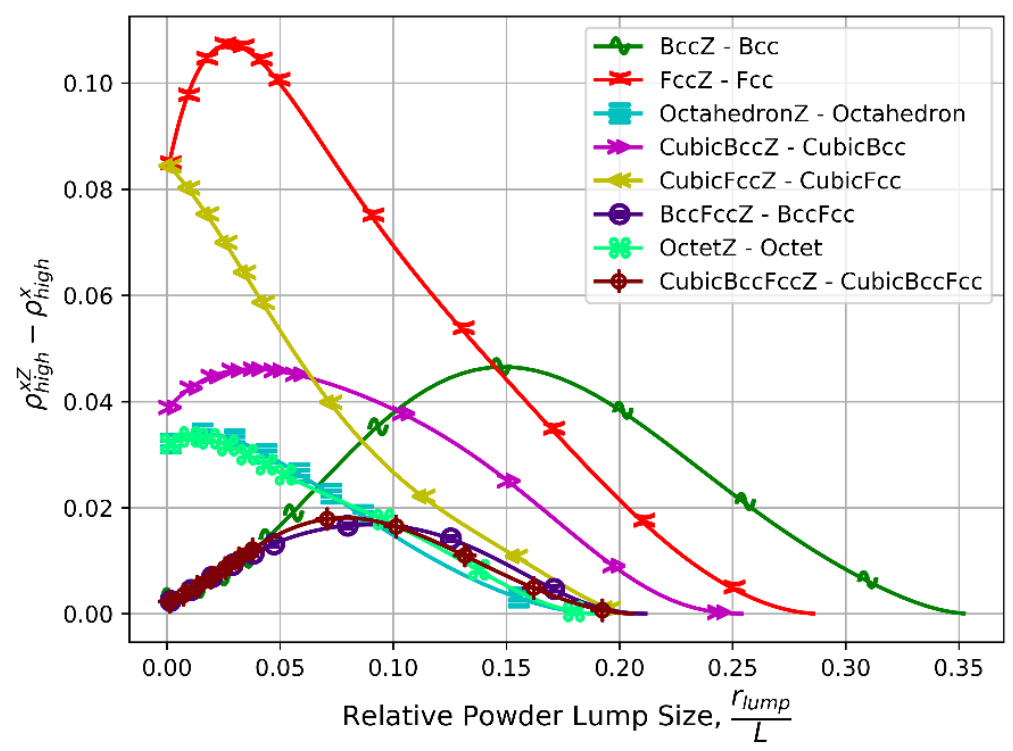

Figure 9. The difference between the higher printable density limits of lattice topologies with and without $Z$-strut in terms of the relative powder lump size

The results showed that introducing Z-strut into the unit-cell shifted both the low and high printable limits upward because the number of struts increased. As the low and the high relative density limits are mutually mirror-symmetric around $\frac{r_{h}^{\prime}}{L}$ in Equation (7) (see Figure 6 also), so are $\rho_{\text {low }}^{x Z}-\rho_{\text {low }}^{x}$ and $\rho_{\text {high }}^{x Z}-\rho_{\text {high }}^{x}$. Yet, $\rho_{\text {low }}^{x Z}-\rho_{\text {low }}^{x}$ and $\rho_{\text {high }}^{x Z}-\rho_{\text {high }}^{x}$ did not have monotonic values. Rather, they varied along with $\frac{r_{\text {min }}}{L}$ and $\frac{r_{l u m p}}{L}$, respectively. This is because their topologies changed, so did their relative density per relative strut radius. Inevitably, the difference would be zero for null and full relative densities. But the interim values would be positive. That is why one sees $\rho_{\text {low }}^{x Z}-\rho_{\text {low }}^{x}$ and $\rho_{\text {high }}^{x Z}-\rho_{\text {high }}^{x}$ curves having concave shapes.

\subsection{Other Parameters Affecting the Printability}

Apart from the parameters affecting the printability which were studied here, strut inclination was a decisive parameter for the printable thickness of a strut $[12,16,17,19,20,45,46]$, which could be found by experiments. But strut inclinations' effect could be treated by artificially worsening the machine precision to find $\rho_{\text {low }}$. Strut inclination also affected the printable length of a strut. The most severe case was, in no doubt, when a strut was oriented horizontally. Struts of $3 \mathrm{~mm}$ in length had been reported as printable by Mazur et al. [17] with SLM. The advancement of technology may improve this limitation. The readers are encouraged to check current state-of-the-art values from the literature before using Figure 6.

Powder adhesion is another problem that needs to be accounted for. Powder lump size can be assumed bigger than its real value. The correction factor should be determined for every material and production method separately. For example, Zhang et al. [22] recommended 3 times bigger lump size. Therefore, a further study focusing on other manufacturing parameters is recommended. This study presented practical estimations for the printable density limits based on powder lump size and machine precision though. As explained in Equation (8), a correction term may be needed

$$
\begin{aligned}
& \rho_{\text {low }}^{\text {real }}=\rho_{\text {low }}+\rho_{\text {low }}^{\text {corr }}=\rho_{\text {low }}\left(\bar{r}+\bar{r}_{\text {corr }}\right) \\
& \rho_{\text {high }}^{\text {real }}=\rho_{\text {high }}-\rho_{\text {high }}^{\text {corr }}=\rho_{\text {high }}\left(\bar{r}-\bar{r}_{\text {corr }}\right) .
\end{aligned}
$$


In order to produce structured-foams with high precision, it is paramount to adopt optimal processing parameters. Hence, finding optimized parameters for each unit-cell will make the range of printable density closer to those discovered in this research.

Lastly, to find the printable density thresholds of the lattices whose struts having cross-sections other than the circular one, this approach can be used by taking $r$ as the radius of the circumscribing circle of that cross-section. However, this approach may behave conservatively for the relative density thresholds.

\section{CONCLUSION}

In this research, direction-wise stretch-dominated strut-based lattice structures were investigated in terms of printable relative volume fraction limits. The printable volume fraction range was mainly controlled by unit-cell size, size of powder lump, and machine precision. The printable density window grew and approached to infinitesimal powder assumption when cell size got bigger while all other parameters remained the same. Contrarily, the printable range narrowed when the machine precision worsened and the powder lump size increased, $c . p$.

Among the lattice types studied, the printable density window of OctahedronZ fell far behind the other unitcell topologies on the high printable limit while BccZ had the biggest window. This pattern was the same as the lattices without Z-struts. Yet the lattices studied here reached higher relative density values, which indicates that these lattice topologies have the advantage of stiffness as well as high relative density limit over the ones without Z-strut. However, their low printable density limit worsened. This meant that the printable density window shifted upward.

Easy-to-use graphs and polynomials for relative density and a nondimensionalized plot to find the printable density window for lattice topologies and size, lump size, and the machine precision were created for the use of designers. To that end, either a software module can be created to check the printability of the lattice design or they can be integrated into a design tool for the user to create a printable lattice structure in the first place.

Further studies focusing on the other process parameters to reach better approximations to the limits are required even though the limits were found, in this work, by accounting two parameters. The real printable density is expected to be closer to the ones found in this study if compensated values for the machine precision and the powder lump are not provided. Advancement in the technology and/or using the best process parameters for the production will make the printable density window closer to the ones provided in this work. Accordingly, this paper is the basis point for further investigations.

\section{CONFLICTS OF INTEREST}

No conflict of interest was declared by the author.

\section{REFERENCES}

[1] Ashby, M. F., "The properties of foams and lattices", Philosophical Transactions of the Royal Society A-Mathematical Physical and Engineering Sciences, 364 (1838): 15-30, (2006).

[2] Rehme, O., Emmelmann, C., "Rapid manufacturing of lattice structures with selective laser melting", In Proceedings of SPIE 6107: 61070K. 1-61070K. 12., (2006).

[3] Sing, S. L., Yeong, W. Y., Wiria, F. E., Tay, B. Y., "Characterization of titanium lattice structures fabricated by selective laser melting using an adapted compressive test method", Experimental Mechanics, 56: 735-748, (2016). 
[4] Loh, L.-E., Chua, C.K., Yeong, W.Y., Song, J., Mapar, M., Sing, S.L., Liu, Z.H., Zhang, D. Q., "Numerical investigation and an effective modelling on the selective laser melting (SLM) process with aluminium alloy 6061”, International Journal of Heat and Mass Transfer, 80: 288-300, (2015).

[5] Tanlak, N., De Lange, D. F., Van, Paepegem W., "Numerical prediction of the printable density range of lattice structures for additive manufacturing", Materials \& Design, 133: 549-558, (2017).

[6] Tanlak, N., "Printable density limits of additively-manufactured structured foams", In Additive Manufacturing Conference Turkey, (2019).

[7] Xu, Y., Zhang, D., Zhou, Y., Wang, W., Cao, X., "Study on topology optimization design, manufacturability, and performance evaluation of Ti-6Al-4V porous structures fabricated by selective laser melting (SLM)", Materials, 10, (2017).

[8] Hedayati, R., Ahmadi, S. M., Lietaert, K., Pouran, B., Li, Y., Weinans, H., Rans, C. D., Zadpoor, A. A., "Isolated and modulated effects of topology and material type on the mechanical properties of additively manufactured porous biomaterials", Journal of the Mechanical Behavior of Biomedical Materials, 79: 254-263, (2018).

[9] Kucewicz, M., Baranowski, P, Małachowski, J, Popławski, A., Płatek, P., "Modelling, and characterization of 3d printed cellular structures", Materials \& Design, 142: 177-189, (2018).

[10] Van Bael, S., Kerckhofs, G., Moesen, M., Pyka, G., Schrooten, J., Kruth, J. P., "Micro-ct-based improvement of geometrical and mechanical controllability of selective laser melted ti6al4v porous structures", Materials Science and Engineering: A, 528(24): 7423-7431, (2011).

[11] Hao, L., Raymont, D., Yan, C., Hussein, A., Young, P., "Design and additive manufacturing of cellular lattice structures", Innovative Developments in Virtual and Physical Prototyping, 249-254, (2011).

[12] Drescher, P, Reimann, T., Seitz, H., "Investigation of powder removal of net-structured titanium parts made from electron beam melting", International Journal of Rapid Manufacturing, 4(2), (2014).

[13] Yan, X., Li, Q., Yin, S., Chen, Z., Jenkins, R., Chen, C., Wang, J., Ma, W., Bolot, R., Lupoi, R., Ren, Z., Liao, H., Liu, M., "Mechanical and in vitro study of an isotropic ti6al4v lattice structure fabricated using selective laser melting", Journal of Alloys and Compounds, 782: 209-223, (2019).

[14] Kim, T. B., Yue, S., Zhang, Z., Jones, E., Jones, J. R., Lee, P. D., “Additive manufactured porous titanium structures: Through-process quantification of pore and strut networks", Journal of Materials Processing Technology, 214 (11): 2706-2715, (2014).

[15] Abele, E., Stoffregen, H. A., Klimkeit, K., Hoche, H., Oechsner, M., "Optimisation of process parameters for lattice structures", Rapid Prototyping Journal, 21(1): 117-127, (2015).

[16] Leary, M., Mazur, M., Elambasseril, J., McMillan, M., Chirent, T., Suna, Y., Qian, M., Eastona, M., Brandt, M., "Selective laser melting (SLM) of AlSi12Mg lattice structures", Materials and Design, 98: 344-357, (2016).

[17] Mazur, M., Leary, M., Sun, S., Vcelka, M., Shidid, D., Brandt, M., "Deformation and failure behaviour of Ti-6Al-4V lattice structures manufactured by selective laser melting (SLM)", The International Journal of Advanced Manufacturing Technology, 84: 1391-1411, (2016).

[18] Wang, D., Wu, S., Fu, F., Mai, S., Yang, Y., Liu, Y., Song, C., "Mechanisms and characteristics of spatter generation in $\{\mathrm{SLM}\}$ processing and its effect on the properties", Materials \& Design, 117: 121-130, (2017). 
[19] Weißmann, V., Drescher, P., Bader, R., Seitz, H., Hansmann, H., Laufer, N., "Comparison of single Ti6A14V struts made using selective laser melting and electron beam melting subject to part orientation", Metals, 7(3): 91-113, (2017).

[20] Fox, J. C., Moylan, S. P., Lane, B. M., "Effect of process parameters on the surface roughness of overhanging structures in laser powder bed fusion additive manufacturing", Procedia CIRP, 45: 131134, (2016).

[21] Spierings, A., Herres, N., Levy, G., "Influence of the particle size distribution on surface quality and mechanical properties in am steel parts", Rapid Prototyping Journal, 17(3): 195-202, (2011).

[22] Zhang, S., Wei, Q., Cheng, L., "Effects of scan line spacing on pore characteristic and mechanical properties of porous Ti6Al4V implants fabricated by selective laser melting", Materials Design, 63: 185-93, (2014).

[23] Yan, C., Hao, L., Hussein A., Young P., Raymont D., "Advanced lightweight 316L stainless steel cellular lattice structures fabricated via selective laser melting", Materials \& Design, 55: 533-541, (2014).

[24] Hazlehurst, K., Wang, C. J., Stanford, M., "Evaluation of the stiffness characteristics of square pore cocrmo cellular structures manufactured using laser melting technology for potential orthopaedic applications", Materials Design, 51: 949-955, (2013).

[25] Tsopanos, S., Mines, R. A. W., McKown, S., Shen, Y., Cantwell, W. J., Brooks, W., Sutcliffe, C. J., "The influence of processing parameters on the mechanical properties of selectively laser melted stainless steel microlattice structures", Journal of Manufacturing Science and Engineering, 132 (041011), (2010).

[26] Vyatskikh, A., Delalande, S., Kudo, A., Zhang, X., Portela, C. M., Greer, J. R., "Additive manufacturing of 3D nano-architected metals", Nature Communications, 9, (593), (2018).

[27] Pattanayak, D. K., Matsushita, T., Takadama, H., Fukuda, A., Takemoto, M., Fujibayashi, S., Sasaki, K., Nishida, N., Nakamura, T., Kokubo, T., "Fabrication of bioactive porous Ti metal with structure similar to human cancellous bone by selective laser melting", Bioceramics Development and Applications, 1: 1-3, (2011).

[28] Qiu, C., Yue, S., Adkins, N.J.E., "Influence of processing conditions on strut structure and compressive properties of cellular lattice structures fabricated by selective laser melting", Materials Science and Engineering: A, 628: 188-97, (2015).

[29] Vrána, R., Koutný, D., Paloušek, D., Pantelejev, L., Jaroš, J., Zikmund, T., Kaiser, J., "Selective laser melting strategy for fabrication of thin struts usable in lattice structures", Materials, 11(9): 1763, (2018).

[30] Sing, S. L., Wiria, F. E., Yeong, W. Y., "Selective laser melting of lattice structures: A statistical approach to manufacturability and mechanical behavior", Robotics and Computer-Integrated Manufacturing, 49: 170-180, (2018).

[31] Salem, H., Carter, L.N., Attallah, M.M., Salem, H.G., "Influence of processing parameters on internal porosity and types of defects formed in Ti6Al4V lattice structure fabricated by selective laser melting", Materials Science and Engineering: A, 767, 11 (2019). 
[32] Yan, C., Hao, L., Hussein, A., Bubb, S. L., Young, P., Raymont, D., "Evaluation of light-weight AlSi10Mg periodic cellular lattice structures fabricated via direct metal laser sintering", Journal of Materials Processing Technology, 214 (4): 856-864, (2014).

[33] Gülcan, O, Konukseven, E. I., Temel, S., "Katmanlı imalatla üretilen Ti6Al4V parçalarının mekanik özellikleri”, Makina Tasarım ve Imalat Dergisi, 15: 27-37, (2017).

[34] Yan, C., Hao, L., Hussein, A., Raymont, D., "Evaluations of cellular lattice structures manufactured using selective laser melting", International Journal of Machine Tools \& Manufacture, 62: 32-38, (2012).

[35] Wauthle, R., Vrancken, B., Beynaerts, B., Jorissen, K., Schrooten, J., Kruth, J.P., Van Humbeeck J., "Effects of build orientation and heat treatment on the microstructure and mechanical properties of selective laser melted Ti6Al4V lattice structures", Additive Manufacturing, 5: 77-84, (2015).

[36] Weißmann, V., Drescher, P., Seitz, H., Hansmann, H., Bader, R., Seyfarth, A., Klinder, A., JonitzHeincke, A., "Effects of build orientation on surface morphology and bone cell activity of additively manufactured Ti6Al4V specimens", Materials, 11 (915), (2018).

[37] Vayre, B., Vignat, F., Villeneuve F., "Identification on some design key parameters for additive manufacturing: Application on electron beam melting", Procedia CIRP, 7: 264-269, (2013).

[38] Chen, W., Watts Seth, Jackson, J. A., Smith, W. L., Tortorelli, D. A., Spadaccini, C. M., "Stiff isotropic lattices beyond the maxwell criterion", Science Advances, 5(9), (2019).

[39] J. C. Maxwell F.R.S. L., "On the calculation of the equilibrium and stiffness of frames", The London, Edinburgh, and Dublin Philosophical Magazine and Journal of Science, 27 (182): 294-299, (1864).

[40] Calladine, C. R., "Theory of shell structures, chapter Streching and bending in cylindrical and nearlycylindrical shells", Cambridge University Press, 238-240, (1983).

[41] Pellegrino, S., Calladine, C. R., "Matrix analysis of statically and kinematically indeterminate frameworks", International Journal of Solids and Structures, 22 (4): 409-428, (1986).

[42] Köhnen, P., Haase, C., Bültmann, J., Ziegler, S., Schleifenbaum, J. H., Bleck, W., "Mechanical properties and deformation behavior of additively manufactured lattice structures of stainless steel", Materials \& Design, 145: 205-217, (2018).

[43] Maskery, I., Sturm, L., Aremu, A. O., Panesar, A., Williams, C. B., Tuck, C. J., Wildman, R. D., Ashcroft, I. A., Hague, R. J. M., "Insights into the mechanical properties of several triply periodic minimal surface lattice structures made by polymer additive manufacturing", Polymer, 152: 62-71, 2018.

[44] Abaqus CAE User’s Guide, Providence, RI, USA. (2018).

[45] Triantaphyllou, A., Giusca, C. L, Macaulay, G. D., Roerig, F., Hoebel, M., Leach, R. K., Tomita, B., Milne, K. A., "Surface texture measurement for additive manufacturing", Surface Topography: Metrology and Properties, 3 (2): 024002, (2015).

[46] Suard, M., Martin, G., Lhuissier, P., Dendievel, R., Vignat, F., Blandin, J. J., Villeneuve, F., "Mechanical equivalent diameter of single struts for the stiffness prediction of lattice structures produced by electron beam melting", Additive Manufacturing, 8: 124-131, (2015). 